\title{
Photon-Photon luminosities in relativistic heavy ion collisions at LHC energies
}

\author{
Kai Hencken, Dirk Trautmann \\ Institut für theoretische Physik der Universität Basel, Klingelbergstrasse 82, 4056 Basel, \\ Switzerland \\ Gerhard Baur \\ Institut für Kernphysik (Theorie), Forschungszentrum Jülich, 52425 Jülich, Germany
}

(March 16, 1995)

\begin{abstract}
Effective $\gamma-\gamma$ luminosities are calculated for various realistic hadron collider scenarios. The main characteristics of photon-photon processes at relativistic heavy-ion colliders are established and compared to the corresponding $\gamma-\gamma$ luminosities at $e^{+}-e^{-}$- and future Photon Linear Colliders (PLC). Higher order corrections as well as inelastic processes are discussed. It is concluded that feasible high luminosity Ca-Ca collisions at the Large Hadron Collider (LHC) are an interesting option for $\gamma-\gamma$ physics up to about $100 \mathrm{GeV} \gamma-\gamma \mathrm{CM}$ energy.

25.75.+r,13.40.-f
\end{abstract}

Typeset using REVTEX 


\section{INTRODUCTION}

Due to the coherent action of $Z$ protons, strong electromagnetic pulses of short duration are produced in relativistic heavy-ion collisions. Among other things, this can be useful for the study of photon-photon collisions. Up to now, photon-photon collisions have been mainly studied at $e^{+} e^{-}$colliders [1 [3]. In contrast to the pointlike electrons and positrons, nuclei are extended objects with an internal structure and a size given by the radius $R \approx$ $1.2 A^{1 / 3} \mathrm{fm}, A$ being the mass number. Also, the strong interaction between the nuclei in central collisions leads to a lower cutoff of useful impact parameters at $b \approx 2 R$. In the last years the study of $\gamma-\gamma$-interactions in heavy-ion collisions has been pursued by many groups. We refer to Refs. [⿴囗大 5 .5 for a list of further references. The spacelike virtual photon, which is emitted by each nucleus, can be considered as quasireal. An exception is the production of

$e^{+} e^{-}$pairs [6]; in this case, the mass of the electron $m_{e}$ is small compared to the maximum value of $\sqrt{\left|q^{2}\right|}$, which is of the order of $1 / R$. Thus, generally, we can calculate $\gamma-\gamma$ cross sections in RHI collisions in the factorized form (see also Fig. 四)

$$
\frac{d \sigma}{d \Omega}(A A \rightarrow A A X)=\int d W \frac{d L_{\gamma \gamma}}{d W} \frac{d \sigma}{d \Omega}(\gamma \gamma \rightarrow X),
$$

where $W$ is the invariant mass of the photon-photon system. The effective $\gamma$ - $\gamma$-luminosity $d \tilde{L}_{\gamma \gamma} / d W$ is given by

$$
\frac{d \tilde{L}_{\gamma \gamma}}{d W}=\frac{d L_{\gamma \gamma}}{d W} L_{A A}
$$

where $L_{A A}$ is the $A-A$ luminosity. Due to the coherence of the photon emission, there is a $Z^{4}$ factor for the $\gamma-\gamma$ cross section. This factor is partly compensated by the lower luminosity achieved for the heavy ions, as compared to the $p-p$ case.

In Sec. [1] effective $\gamma-\gamma$ luminosities are compared with each other for various collider types. We use the luminosities of the heavy-ion beams from a recent study by Eggert and Morsch [7,8]. Unrestricted, as well as restricted (in the rapidity $Y$ ) $\gamma-\gamma$ luminosities are discussed. This extends the work of Ref. [4]. In Sec. III we study the influence of higher order processes and the effects of inelastic processes. Inelastic processes are those, where the emission of the equivalent photon leads to a nuclear transition. Of special interest is the case, where the nucleus makes a transition to the giant dipole resonance (GDR), a very collective nuclear mode. The study of such kind of effects is important for a detailed planning and optimization of the experiments, see e. g. [9. 10]. A more qualitative comparison to the case of $\gamma-\gamma$-physics in $p-p$ collisions is also made. Our conclusions are given in Sec. IV. In the appendix we give a short derivation of the inelastic double equivalent photon approximation (DEPA) and apply it to the nuclear GDR excitation.

\section{EFFECTIVE $\gamma-\gamma$-LUMINOSITIES FOR HADRON- AND $e^{+} e^{-}$-COLLIDERS}

In this section we are going to compare the effective $\gamma-\gamma$ luminosities for $\mathrm{Ca}-\mathrm{Ca}$ and $\mathrm{Pb}-\mathrm{Pb}$ beams at the LHC with those of other colliders. We want to study whether the heavy-ion beams can compete with other colliders. 
We calculate the $\gamma-\gamma$ luminosities in the heavy-ion case using the semiclassical impactparameter-dependent form of the photon density, as described in [11,12]. The $b$-dependent equivalent photon number is given by

$$
N(\omega, b)=\frac{Z^{2} \alpha}{\pi^{2}}\left(\frac{\omega^{2}}{\gamma^{2}}\right) K_{1}^{2}\left(\frac{\omega b}{\gamma}\right),
$$

where $K_{1}$ is the modified Bessel function of the second kind. The total unrestricted $\gamma-\gamma$ luminosity is then given by [11,13.

$$
\begin{aligned}
d L / d W= & \frac{4 \pi}{W} \int d Y \int_{R_{1}}^{\infty} b_{1} d b_{1} \int_{R_{2}}^{\infty} b_{2} d b_{2} \int_{0}^{2 \pi} d \phi N\left(\frac{W}{2} e^{Y}, b_{1}\right) N\left(\frac{W}{2} e^{-Y}, b_{2}\right) \\
& \Theta\left(b_{1}^{2}+b_{2}^{2}-b_{1} b_{2} \cos (\phi)-\left(R_{1}+R_{2}\right)\right),
\end{aligned}
$$

where we have introduced a cutoff in the impact parameter, in order to discriminate for central collisions, where both ions interact strongly with each other. In order to have a clean signal, we drop these events. Due to this cutoff, we do not need a form factor explicitly. For $p-p$ and $e^{+}-e^{-}$collisions we use the formulae from the literature [14,2] (For the $p$ - $p$ case only the elastic part of the luminosity is used.)

$$
\begin{aligned}
d L / d W(p p) & =\frac{2 W}{s} \frac{1}{\tau}\left(\frac{\alpha}{\pi}\right)^{2} \frac{2}{3} \log ^{3}\left(\frac{1}{\tau}\right) \\
d L / d W\left(e^{+} e^{-}\right) & =\left(\frac{\alpha}{2 \pi} \log \left(\frac{s}{4 m_{e}^{2}}\right)\right)^{2} \frac{1}{\tau}\left[(2+\tau)^{2} \log \left(\frac{1}{\tau}\right)-2(1-\tau)(3+\tau)\right],
\end{aligned}
$$

with $\tau=W^{2} / s$ and $s=\left(P_{1}+P_{2}\right)^{2}$. In the $e^{+}-e^{-}$case the formula also includes correction due to the collisions, where the lepton can loose an appreciable part of its energy. For the next generation of photon-photon colliders, which are based on the Compton backscattering of an electron beam in an intense laser beam [15], we use the results of Fig. 9 of Ref. [16]. Here we use the result for the "conversion distance" $z=0 \mathrm{~cm}$, where the photon luminosity is maximal.

In Fig. 2 we compare the unrestricted luminosities of these colliders. The different beam luminosities and energies used in the comparison are summarized in Table $\mathbb{1}$. We see that the spectrum for $p-p$ collisions is much harder for higher invariant masses than that for heavy ions. This is due to the smaller radius of the proton. For small values of $W$ the different luminosities are mainly proportional to the beam luminosity and $Z^{4}$. For small enough $W$, the $\gamma$ dependence is given by 17,18

$$
d L / d W=\left(\frac{Z^{2} \alpha}{\pi}\right)^{2} \frac{32}{3 W} \ln ^{3}\left(\frac{2 \gamma}{W R}\right) .
$$

For the LHC, we see that the Ca-Ca collisions seem to be the desired choice, whereas the $\mathrm{Pb}-\mathrm{Pb}$ case is even lower than the $p$ - $p$ case for higher invariant masses.

The planned PLC would be superior, as a dedicated photon-photon machine, for $W>$ $40 \mathrm{GeV}$; its energy range exceeds what will be possible with the LHC. Please note that the photon-spectrum shown in the figure is only the low energy part of it. Most photons are produced with energies in the TeV region. But whereas the LHC is accepted now and will 
be in operation in 2004 or $2008, \gamma-\gamma$ colliders are only in a planning stage. Therefore, we think that photon-photon physics at the LHC is an interesting option, extending the range of the invariant mass to energies not possible today.

As already discussed in [4], the unrestricted luminosity is not the one relevant directly for experiments. Depending on the detectors used, only a part of all photon-photon processes may be detected. Which part of the processes are really observed, depends on the detector system and on the produced particles. The importance of such a cutoff can be demonstrated, by using a simple formula for a cutoff [2]

$$
\left|p_{\gamma \gamma}\right|<f W
$$

Setting $f=1$, we get a restriction on the $Y$ range with $-Y_{0}<Y<Y_{0}$ and

$$
Y_{0}=\ln (1+\sqrt{2})
$$

In Fig. 3 we compare the restricted luminosities with the unrestricted ones for $\mathrm{Ca}-\mathrm{Ca}$ and $\mathrm{Pb}-\mathrm{Pb}$. We see that they are reduced for small invariant mass as expected [4].

Due to the factorization property of Eq. (1), these luminosities can now be used easily in order to calculate the different $\gamma-\gamma$ processes, leading to the production of resonances and continuum states. For this we need the cross sections for the photon-photon subprocess. We refer here to the large literature on this subject, especially the production of heavy quark systems, the production of the Higgs boson and of supersymmetric particles [9, 14, 19].

\section{HIGHER ORDER AND INELASTIC PROCESSES}

In contrast to the electron and positrons, nuclei are extended objects with a rich excitation spectrum. Now in connection with the planning of the experiments, the question arises how this nuclear structure influences the $\gamma-\gamma$ spectrum. In Section $\mathbb{I}$ the effect of the finite size of the nucleus is fully accounted for by the introduction of the cutoff $b_{\min }=2 R\left(b_{1}>R\right.$, $b_{2}>R$ ) in impact parameter space, i.e., the elastic charge form-factor of the nucleus is included.

In the following we consider two different effects, which lead to excited nuclear states. Firstly a higher order electromagnetic process where there is, in addition to the $\gamma$ - $\gamma$ process, an inelastic $\gamma A$ interaction. The process is shown in Fig. 14 . The two different processes can be seen as independent, if we assume, that the elastic form factor, which is determined by the size of the system, remains nearly the same. Then it is simplest to adopt the semiclassical impact parameter approach. The probability for a $\gamma \gamma \rightarrow f$ process and the $\gamma A \rightarrow A^{*}$ process is given by the product

$$
P_{f A^{*}}(b)=P_{\gamma \gamma \rightarrow f}(b) P_{\gamma A \rightarrow A^{*}}(b) .
$$

Integrating from $b=2 R$ up to infinity, we obtain for the cross section for $\gamma \gamma \rightarrow f$ fusion accompanied by a specific $\gamma A \rightarrow A^{*}$ interaction

$$
\sigma_{f A^{*}}=2 \pi \int_{2 R}^{\infty} b d b P_{\gamma \gamma \rightarrow f}(b) P_{\gamma A \rightarrow A^{*}}(b) .
$$

Since $\sum_{A^{*}} P_{\gamma A \rightarrow A^{*}}=1$ (where the sum over $A^{*}$ includes the ground state also), we have 


$$
\sum_{A^{*}} \sigma_{f A^{*}}=\sigma_{f}=2 \pi \int_{2 R}^{\infty} b d b P_{\gamma \gamma \rightarrow f}(b)
$$

$\sigma_{f}$ is the cross section of the $\gamma \gamma \rightarrow f$ process alone, without any higher order processes, which was calculated up to now. We define $\sigma_{f, A}$ as the cross section for a $\gamma \gamma \rightarrow f$ process, not accompanied by a $\gamma A$ interaction, and $\sigma_{f, A^{*}}$, where such an interaction takes place. An estimate can be given using the following argument: Usually the integrand will be peaked at $b \approx b_{\min }=2 R$, and we approximate therefore

$$
\sigma_{f, A} \approx 2 \pi P_{A}(2 R) \int_{2 R}^{\infty} b d b P_{\gamma \gamma \rightarrow f}(b)=2 \pi P_{A}(2 R) \sigma_{\gamma \gamma \rightarrow f},
$$

and

$$
\sigma_{f, A^{*}} \approx 2 \pi P_{A^{*}}(2 R) \int_{2 R}^{\infty} b d b P_{\gamma \gamma \rightarrow f}(b)=2 \pi P_{A^{*}}(2 R) \sigma_{\gamma \gamma \rightarrow f},
$$

where $P_{A}+P_{A^{*}}=1$ and $P_{A^{*}}=\sum_{A^{*} \neq A} P_{\gamma A \rightarrow A^{*}}$.

Impact parameter dependent probabilities were calculated in $4,18,20]$. They depend strongly on $A$. Especially important is the excitation of the giant dipole resonance (GDR), which is assumed to be located at

$$
E_{G D R}=80 \mathrm{MeV} \frac{1}{A^{1 / 3}} .
$$

The excitation probability can be calculated, assuming that it has zero decay width and that it exhausts the Thomas-Reiche-Kuhn (TRK) sum rule. As the probability for this excitation becomes large for $\mathrm{Pb}-\mathrm{Pb}$-collisions, we include in our calculation the effects of multiphonon excitation. The sum over all these excitations is given by (see Eq. (3.2.4) of [18)

$$
\begin{aligned}
P_{G D R}(b) & =1-\exp \left(-\frac{Z^{2} \alpha}{E_{G D R} \pi^{2}} \frac{1}{b^{2}} \int_{0}^{\infty} \frac{d \omega}{\omega} \sigma_{\gamma A}(\omega)\right) \\
& \approx 1-\exp \left(-\frac{Z^{2} \alpha}{E_{G D R} \pi^{2}} \frac{1}{b^{2}} 60 \frac{N Z}{A} \mathrm{MeV} m b\right) .
\end{aligned}
$$

This gives an excitation probability of about $40 \%$ for $\mathrm{Pb}$ and of about $1 \%$ for $\mathrm{Ca}$ at $b=2 R$. As each of the ions can be excited, we get a total probability for the excitation of at least one of them of about $2 \%$ for $\mathrm{Ca}$ and of about $65 \%$ for $\mathrm{Pb}$. With less probability the quasideuteron region, the nucleon resonances and also the nuclear continuum are excited. While these effects are appreciable for the $\mathrm{Pb}-\mathrm{Pb}$ case, the effects are only of minor importance for lighter systems like $\mathrm{Ca}-\mathrm{Ca}$, due to the strong $Z$ dependence.

A detailed calculation of the cross section using the $b$ dependent probability as given by Eqs. (15) and (16) and integrating numerically over the impact parameter gives the results of Fig. 5. These are in qualitative agreement with Eq. (14). Whereas Eq. (14) is too high for small invariant masses, it gives the right order of magnitude for large ones. This is due to the fact, that processes with small invariant mass are possible at larger impact parameter, whereas high invariant masses are possible only for close collisions.

Most frequently the excitation of the GDR state is followed by neutron evaporation. This leads to a change of the mass to charge ratio of the nucleus and the fragments will be lost from the circulating beam and can possibly be detected in a zero degree calorimeter (ZDC). 
In addition to the elastic EPA of Sec. II], where the nucleus remains in its ground state during the photon emission process, there are also elastic-inelastic and even inelastic-inelastic processes, where the emission of the photon leads to the excitation of the nucleus. The corresponding situation was studied by Ohnemus et al. [14] for $p$ - $p$ collisions, who found that inelastic processes are a non-negligible contribution in this case. Let us say a few words about the qualitative differences between the $p-p$ and $A$ - $A$ case. A typical inelastic-elastic process is shown in Fig. 6. In the appendix, we give a short derivation of the generalization of the elastic EPA to inelastic processes. On the one hand, we get a modification of the formula due to the different vertex factor, on the other hand, the minimal $q^{2}$ is modified as we have an inelastic process. For the most important case of the GDR excitation the spectrum of the equivalent photons is also given in the appendix. As demonstrated there, the spectrum is much more sensitive to the detailed form of the form factor in the inelastic case as in the elastic case. Therefore we have used two different form factors, a dipole form factor given by

$$
F\left(k^{2}\right)=\frac{\Lambda^{4}}{\left(\Lambda^{2}+k^{2}\right)^{2}},
$$

and a Gaussian form factor

$$
F\left(k^{2}\right)=\exp \left(-\frac{k^{2}}{\lambda^{2}}\right)
$$

Integrating over $\omega$, we get the luminosities for the elastic-inelastic processes as shown in Figs. 7 and 8. The contribution of the elastic-inelastic processes are about 1\%, so the detailed form of the form factor is not important in this case.

Inelastic processes are only a small correction. This can be understood from the fact, that the electromagnetic transition current remains finite for $q^{2} \rightarrow 0$ only in the elastic case. For inelastic processes it vanishes at least with $q$, compensating therefore the enhancement of the process due to the small $q^{2}$ in the photon propagator.

Another process, which has to be considered, is the incoherent scattering of an individual proton instead of the whole nucleus (see Fig. 9), where $q^{2}$ is larger than $1 / R^{2}$. It seems interesting to compare the elastic and inelastic contributions to the equivalent photon spectrum in the $p$ - $p$ and $A$-A-cases (see also [14]). We consider a proton as being made up of $u$ and $d$ quarks, with charges $2 / 3$ and $-1 / 3$, respectively. A nucleus is made up of $Z$ protons, which are then made up of quarks. Whereas the charge of the proton is comparable to the charge of its individual constituents, the charge of the nucleus is much larger than the charge of its constituents, if $Z \gg 1$. Thus for nuclei, the equivalent photon spectrum is dominated strongly by the coherent component, where $q^{2} \ll 1 / R^{2}$, whereas for protons the individual, incoherent quark contributions are comparable, or even larger, than the elastic component. This is in accordance with the quantitative results of Ref. [14].

It has been suggested [8] to use $\mu^{+} \mu^{-}$-pair-production as a luminosity monitor for the $p-p$ and the $A$ - $A$ collider. The $\gamma-\gamma$ mechanism dominates for collisions with no strong interactions between the protons. This condition can be assured experimentally. In addition to the elastic and incoherent contributions, also the transition to nucleon resonances, notably the $\Delta$, have to be taken into account. Since the relevant electromagnetic form factors are known, a reliable calculation of the $\mu^{+} \mu^{-}$production via the $\gamma-\gamma$ mechanism should be feasible. The 
same formalism, which was used here for the GDR excitation of a nucleus, can also be used for this case. This is the subject of future work, beyond the scope of the present paper.

\section{CONCLUSIONS}

The possibility to do photon-photon physics with heavy ions at LHC energies was scrutinized. We calculate unrestricted and restricted luminosities for $\mathrm{Pb}$ and $\mathrm{Ca}$. For large invariant masses heavy ions notably $\mathrm{Ca}-\mathrm{Ca}$, with its relatively high luminosity, compare very favorably with LEP200, e.g.. For very high invariant masses, $W \gtrsim 200 \mathrm{GeV}$, the $p-p$ option becomes better; this is essentially due to the harder form factor of the proton as compared to the softer nuclear form factor. In the far future a dedicated photon-photon collider (like PLC) will have a larger $\gamma-\gamma$ luminosity than the $A A$ options over most of the interesting invariant mass regions.

Also with the aim to help planning experiments, higher order and other possibly important correction factors were investigated. We find that for very high $Z$, like $\mathrm{Pb}-\mathrm{Pb}$, most of the $\gamma-\gamma$ collisions are accompanied by a nuclear excitation, predominantly GDR excitation. For the $\mathrm{Ca}-\mathrm{Ca}$ case such effects are almost negligible. We show that in contrast to the $p-p$ case $\gamma-\gamma$ events from inelastic photon emission are of minor importance for $A A$ collisions. We conclude that $\gamma-\gamma$ physics at the LHC looks promising. Of course, more detailed studies, also of the experimental design, will be necessary.

\section{ACKNOWLEDGMENT}

It is a pleasure to thank K. Eggert, H. Gutbrod, A. Morsch, S. Sadovsky, and J. Schukraft for their helpful, encouraging and stimulating comments.

\section{APPENDIX: THE DOUBLE EQUIVALENT PHOTON APPROXIMATION FOR INELASTIC PROCESSES}

Our derivation for the equivalent photon approximation generalized to inelastic processes follows in principle that of [3] (using a slightly different definition of $\rho_{\mu \nu}$ ). In this short derivation we will not discuss the general applicability of the EPA, but safely neglect the contribution of the longitudinal photons in comparison to the transversal photons. For a detailed discussion of the applicability see, e.g., [3].

The cross section for the process (see also Fig. 6) is given by

$$
d \sigma_{A A}=(2 \pi)^{4} \delta\left(P_{f}+P_{1}^{\prime}-P_{1}+P_{2}^{\prime}-P_{2}\right)|M|^{2} \frac{1}{4 I} \frac{d^{3} p_{1}^{\prime}}{(2 \pi)^{3} 2 E_{1}^{\prime}} \frac{d^{3} p_{2}^{\prime}}{(2 \pi)^{3} 2 E_{2}^{\prime}} d \Gamma,
$$

where we do not make any assumptions on projectile and target and also on the produced system $f . P_{f}$ is the total momentum of this system $f, d \Gamma$ its phase space. $|M|^{2}$ is given by

$$
|M|^{2}=\frac{1}{\left(q_{1}^{2}\right)^{2}} \frac{1}{\left(q_{2}^{2}\right)^{2}} \Gamma_{1}^{\mu} \Gamma_{1}^{\mu^{\prime} *} \Gamma_{2}^{\nu} \Gamma_{2}^{\nu^{\prime} *} W_{\mu \nu \mu^{\prime} \nu^{\prime}}
$$


with the momentum of the virtual photon $q_{i}=P_{i}^{\prime}-P_{i}$ and the electromagnetic transition current $\Gamma_{i}$. In the EPA we neglect the dependence of $W_{\mu \nu \mu^{\prime} \nu^{\prime}}$ on $q_{i}^{2}$ and replace it with the one for real photons. Therefore $W_{\mu \nu \mu^{\prime} \nu^{\prime}}$ depends only on $\omega_{1}$ and $\omega_{2}$. Averaging over the initial state and summing over the final state, we get the "photon densities" $\rho_{A}$

$$
\rho_{A}^{\mu \nu}:=\frac{1}{2 J_{i}+1} \sum_{M_{i}, M_{f}} \Gamma^{\mu} \Gamma^{\nu *} .
$$

It is well known from general invariance considerations that the general form of $\rho_{A}$ for an arbitrary transition has to be of the form

$$
\rho_{A}^{\mu \nu}=\left(g^{\mu \nu}-\frac{q^{\mu} q^{\nu}}{q^{2}}\right) C+\left(P^{\mu}-\frac{q P}{q^{2}} q^{\mu}\right)\left(P^{\nu}-\frac{q P}{q^{2}} q^{\nu}\right) D,
$$

where $C$ and $D$ are function of invariants only.

If the particle is moving relativistically, the photon momentum is almost aligned to the beam axis. In the EPA, we need only the transverse components of $\rho$, transverse with respect to $q$. In the relativistic case, these transverse components are given by

$$
\left\langle\rho_{t}^{\mu \nu}\right\rangle=\left(-2 C+D \frac{q_{\perp}^{2}}{\omega^{2}} P^{2}\right) \frac{1}{2} \sum_{\lambda} \epsilon_{\lambda}^{\mu} \epsilon_{\lambda}^{\nu *},
$$

where $P=m \beta \gamma$ is the absolut value of the spatial components of the heavy ion momentum and we have already averaged over the transverse plane, which is normally not measured. The $\epsilon_{\lambda}$ are the two transverse polarizations of a corresponding real photon. We see that the photon density is proportional to the unpolarized real photon density. Using the formula for the real photon-photon cross section, we can write the cross section integrated over the scattered particles as

$$
d \sigma_{A A}=\int \frac{d \omega_{1}}{\omega_{1}} \frac{d \omega_{2}}{\omega_{2}} N_{1}\left(\omega_{1}\right) N_{2}\left(\omega_{2}\right) d \sigma_{\gamma \gamma}\left(\omega_{1}, \omega_{2}\right)
$$

with the equivalent photon numbers $N_{i}\left(\omega_{i}\right),(i=1,2)$ given by

$$
N_{i}\left(\omega_{i}\right)=\int \frac{\left(-2 C_{i}+q_{i \perp}^{2} / \omega_{i}^{2} P_{i}^{2} D_{i}\right) \omega_{i}^{2}}{(2 \pi)^{3} 2 E_{i} P_{i}\left(q_{i}^{2}\right)^{2}} d^{2} q_{i \perp} .
$$

For the elastic collision of a spinless particle we have $C=0$ and $D=4|F|^{2}$, where $F$ is the elastic form factor. $N(\omega)$ is then given by

$$
N(\omega)=\int \frac{2 F^{2}\left(q^{2}\right) q_{\perp}^{2}}{(2 \pi)^{3}\left(\omega^{2} / \gamma^{2}+q_{\perp}^{2}\right)^{2}} 2 \pi q_{\perp} d q_{\perp},
$$

where we have used the fact, that for an elastic collision $q^{2}=-\left(\omega^{2} / \gamma^{2}+q_{\perp}^{2}\right)$. Using a simple form factor $F^{2}=4 \pi Z^{2} \alpha$ and integrating over $q_{\perp}$ from 0 to a cut-off value $\lambda=1 / R$, we get the EPA spectrum in the leading order as

$$
N(\omega)=\frac{2 Z^{2} \alpha}{\pi} \ln \left(\frac{\gamma}{\omega R}\right)
$$


This is just the standard result [3, 18].

In order to find the corresponding expressions for $C$ and $D$ for a photon emission with nuclear excitation, we work in the rest frame of the initial nucleus and write $C$ and $D$ in invariant form. In order to distinguish between both systems, we write the photon momentum in the rest frame as $(-\Delta, \vec{k})$. As the expressions normally depend on $|\vec{k}|$, we define this as $k$, in contrast to the Lorentz invariant $q$, which is given by $q^{2}=\Delta^{2}-k^{2}$.

Following deForest-Walecka [21], we can write

$$
\begin{aligned}
\rho^{00} & =4 \pi\left|M^{C}\right|^{2} \\
\rho^{\lambda \lambda^{\prime}} & =\delta_{\lambda, \lambda^{\prime}} 2 \pi\left(\left|T^{e}\right|^{2}+\left|T^{m}\right|^{2}\right) \\
\rho^{0 \lambda} & =0
\end{aligned}
$$

where $\lambda, \lambda^{\prime}$ denotes directions transverse to $\vec{k}$. Together with the connection between $\Gamma^{0}$ and $\vec{\Gamma} \vec{k}$ from the gauge invariance

$$
\Delta \Gamma^{0}=-\vec{k} \vec{\Gamma}
$$

this determines all components of the electromagnetic tensor.

Using these equations, $C$ and $D$ can be expressed in terms of $T^{e}, T^{m}$, and $M^{C}[22]$. We get

$$
\begin{aligned}
& C=-2 \pi\left[\left|T^{e}\right|^{2}+\left|T^{m}\right|^{2}\right] \\
& D=\frac{\left(q^{2}\right)^{2}}{k^{4} M^{2}} 2 \pi\left[2\left|M^{C}\right|^{2}-\frac{k^{2}}{q^{2}}\left(\left|T^{e}\right|^{2}+\left|T^{m}\right|^{2}\right)\right] .
\end{aligned}
$$

As a case of special importance, we apply this to the GDR excitation. In the GoldhaberTeller model of the GDR as a harmonic oscillator [21,23], we get

$$
\begin{aligned}
\left|M^{C}\right|^{2} & =4 M^{2}\left(\frac{N}{A}\right)^{2} \frac{k^{2}}{2 \mu} \frac{1}{\Delta} \frac{F^{2}(k)}{4 \pi} \\
\left|T^{e}\right|^{2} & =8 M^{2}\left(\frac{N}{A}\right)^{2} \frac{k^{2}}{2 \mu} \frac{1}{\Delta}\left(\frac{\Delta}{k}\right)^{2} \frac{F^{2}(k)}{4 \pi}
\end{aligned}
$$

where $M$ is the mass of the nucleus and $\mu=(N Z / A) M$. Please note that the form factor $F$ appearing in these equations is the elastic form factor of the nuclei as a function of $k^{2}=\Delta^{2}-q^{2}$. Only in the elastic case are $k^{2}$ and $q^{2}$ identical. Using Eq. (A15) we get

$$
\begin{aligned}
& C=-4 M^{2}\left(\frac{N}{A}\right)^{2} \frac{F^{2}}{2 \mu} \Delta, \\
& D=4\left(\frac{N}{A}\right)^{2} \frac{F^{2}}{2 \mu} \frac{-q^{2}}{\Delta} .
\end{aligned}
$$

The EPA spectrum is then given by

$$
N(\omega)=\int\left(\frac{N}{A}\right)^{2} \frac{4 F^{2}}{2 \mu} \frac{2 M^{2} \Delta^{2} \omega^{2}+q_{\perp}^{2} P^{2}|q|^{2}}{(2 \pi)^{3} 2 E P\left(q^{2}\right)^{2} \Delta} d^{2} q_{\perp} .
$$


In the elastic case, $q^{2}$ was given by $-\left(\omega^{2} / \gamma^{2}+q_{\perp}^{2}\right)$. For the inelastic case, we get for $\gamma \gg 1$

$$
\begin{aligned}
q^{2} & =\Delta^{2}-\left(\frac{\omega}{\gamma \beta}+\frac{\Delta}{\beta}\right)^{2}-q_{\perp}^{2} \\
& \approx-\left[\frac{\omega}{\gamma}\left(\frac{\omega}{\gamma}+2 \Delta\right)+q_{\perp}^{2}\right] .
\end{aligned}
$$

The form factor $F$ appearing in the EPA spectrum is the elastic form factor of the nucleus, written as a function of $k^{2}=\Delta^{2}-q^{2}$. In the elastic case, the spectrum was insensitive to the detailed form of the form factor. Comparing the integrand of Eq. (A8) and Eq. (A20) without a form factor, we see, that for inelastic processes $d N / d q_{\perp}$ increases linearly for large $q_{\perp}$, contrary to the elastic case. In Fig. 10 we compare both in the case of a $\mathrm{Pb}$ nucleus. Therefore the photon spectrum is sensitive to the form factor in the inelastic case. In our calculations we use a dipole form factor

$$
F=\Lambda^{4} /\left(\Lambda^{2}+k^{2}\right)^{2}
$$

and a Gaussian form factor

$$
F=\exp \left(-\frac{k^{2}}{\lambda^{2}}\right),
$$

where the parameters have been chosen to get the right root mean square radius of the nuclei. Integrating over the transverse momentum we get $N(\omega)$ which has been used in order to get the elastic-inelastic $\gamma-\gamma$ luminosity $d L / d W$. 


\section{REFERENCES}

[1] See, e.g., the proceedings of the IVth workshop on Photon-Photon Collisions, UC, San Diego, La Jolla, California, 1992, edited by D. O. Caldwell and H. P. Paar (World Scientific, Singapore, 1992).

[2] F. C. Erné in "ECFA workshop on LEP200", 211 CERN87-08, 1987 (unpublished).

[3] V. M. Budnev, I. F. Ginzburg, G. V. Meledin, V. G. Serbo: Phys. Rep. 15 (1975) 181.

[4] N. Baron, G. Baur: Phys. Rev. C 49 (1994) 1127.

[5] M. Vidović, M. Greiner, Ch. Best, G. Soff: Phys. Rev. C 47 (1993) 2308.

[6] K. Hencken, D. Trautmann, G. Baur: Phys. Rev. A 49 (1994) 1584.

[7] K. Eggert, A. Morsch: LHC Note 264 CERN AT/94-05 (unpublished).

[8] K. Eggert, private communication.

[9] S. Sadovsky: CERN-Note ALICE/93-07 (1993) (unpublished).

[10] "Transparencies of the ALICE workshop" ALICE/MIN 94-05, (1994) (unpublished).

[11] G. Baur, L. G. Ferreira Filho: Nucl. Phys. A 518 (1990) 786.

[12] G. Baur, N. Baron: Nucl. Phys. A 561 (1993) 628.

[13] R. N. Cahn, J. D. Jackson: Phys. Rev. D 42 (1990) 3690.

[14] J. Ohnemus, T. F. Walsh, P. M. Zerwas: Phys. Lett. B 328 (1994) 369.

[15] I. F. Ginzburg: Nucl. Phys. B (Proc. Suppl.) 37B (1994) 303.

[16] D. L. Barden, D. A. Bauer, D. O. Caldwell: SLAC-PUB-5715 (unpublished); see also Phys. Rev. D 48, (1993) 4018.

[17] G. Baur, C. Bertulani: Z. Phys. A 330 (1988) 77.

[18] C.A. Bertulani, G. Baur: Phys. Rep. 163 (1988) 299.

[19] M. Drees, R. M. Godbole, M. Nowakowski, S. D. Rindami: Phys. Rev. D 50 (1994) 2335.

[20] M. Vidović, M. Greiner, G. Soff: Phys. Rev. C 48 (1993) 2011.

[21] T. deForest, J. D. Walecka: Adv. Phys. 15 (1966) 1.

[22] J. D. Walecka: Argonne National Laboratory, ANL-83-50 (1983) (unpublished).

[23] M. Goldhaber, E. Teller: Phys. Rev. 74 (1948) 1046. 


\section{FIGURES}

FIG. 1. General form of an elastic photon-photon process. f denotes the system of the produced particles.

FIG. 2. Comparison of the photon-photon effective luminosity $d \tilde{L}_{\gamma \gamma} / d W$ as a function of the invariant mass $W$ of the produced system. Shown are the results for hadron collider using Ca-Ca (solid line), $\mathrm{Pb}-\mathrm{Pb}$ (dashed line) and $p-p$ collisions (dot-dashed line) together with those of $e^{+}-e^{-}$ (dotted line) and $\gamma-\gamma$ colliders (two-dotted lines) for two different polarizations. See text and Table 1 for the parameter used for the different accelerators.

FIG. 3. Comparison of the unrestricted (solid line) and restricted effective luminosities (dotted line). Shown are the results for Ca-Ca collisions (upper curve) and $\mathrm{Pb}-\mathrm{Pb}$ collisions (lower curve). The Collider parameters used are shown in Table 1 .

FIG. 4. The two processes contributing to the higher order process, where the $\gamma-\gamma$ process accompanied by a nuclear excitation of one of the nuclei.

FIG. 5. Comparison of the total $\gamma-\gamma$ luminosity (dotted line, Eq. (12)), see Eq. (11), with the one, where it is accompanied by GDR excitation of one of the ions (solid line, Eq. (11), where $A^{*}$ is the GDR). Shown are the results for $\mathrm{Pb}-\mathrm{Pb}$ (upper curves) and $\mathrm{Ca}-\mathrm{Ca}$ (lower curves).

FIG. 6. Inelastic-elastic process contributing to the $\gamma-\gamma$ luminosity. In the case of heavy ion collisions, $A^{*}$ denotes an excited state of the nuclei, e.g., a GDR.

FIG. 7. Comparison of the contributions of the elastic-elastic and inelastic-elastic processes to the $\gamma-\gamma$ luminosity for a $\mathrm{Pb}-\mathrm{Pb}$ collision. Shown are the results for elastic-elastic process (dotted

line) together with the inelastic-elastic processes using a Gauss form factor (solid line) and a dipole form factor (dashed line).

FIG. 8. Same as Fig. 7 but now for a Ca-Ca collision.

FIG. 9. Inelastic Photon-photon process due to the photon emission of an individual "parton" as opposed to the whole particle. For heavy ions we take the partons to be the protons, for $p-p$ collisions the quarks.

FIG. 10. The differential photon number $d N(\omega) / d q_{\perp}$ is compared for a $\mathrm{Pb}-\mathrm{Pb}$ collision and $\omega=10 \mathrm{GeV}$ for elastic (dotted line, Eq. (A8)) and inelastic photon emission (solid line, Eq. (A20)). The vertical line shows the range of $q_{\perp}$, which is limited due to the form factor. The result of the inelastic case has been multiplied by a factor of 400 . 


\section{TABLES}

\begin{tabular}{lcrr}
\hline \hline Collider & Luminosity $\left(\mathrm{cm}^{-2} \mathrm{~s}^{-1}\right)$ & $E_{c m}$ & $\gamma$ \\
\hline $\mathrm{LEP} 200$ & $1 \times 10^{32}$ & $100 \mathrm{GeV}$ & $2 \times 10^{5}$ \\
$p$ - $p$ at LHC & $1 \times 10^{33}$ & $7 \mathrm{TeV}$ & 7500 \\
$\mathrm{~Pb}-\mathrm{Pb}$ at LHC & $5 \times 10^{26}$ & $7 \mathrm{ATeV} Z / A$ & 2900 \\
$\mathrm{Ca}-\mathrm{Ca}$ at LHC & $5 \times 10^{30}$ & $7 \mathrm{ATeV} Z / A$ & 3700 \\
$\mathrm{NLC} / \mathrm{PLC}(z=0 \mathrm{~cm})$ & $2 \times 10^{33}$ & $500 \mathrm{GeV}$ & \\
\hline \hline
\end{tabular}

TABLE I. The collider parameters used in the comparison of the different $\gamma-\gamma$ luminosities. Please see text for References of the different colliders and explanation of $z$. 
This figure "fig1-1.png" is available in "png" format from: http://arxiv.org/ps/nucl-th/9410014v2 
This figure "fig1-2.png" is available in "png" format from: http://arxiv.org/ps/nucl-th/9410014v2 
This figure "fig1-3.png" is available in "png" format from: http://arxiv.org/ps/nucl-th/9410014v2 
This figure "fig1-4.png" is available in "png" format from: http://arxiv.org/ps/nucl-th/9410014v2 
This figure "fig1-5.png" is available in "png" format from: http://arxiv.org/ps/nucl-th/9410014v2 
This figure "fig1-6.png" is available in "png" format from: http://arxiv.org/ps/nucl-th/9410014v2 
This figure "fig1-7.png" is available in "png" format from: http://arxiv.org/ps/nucl-th/9410014v2 
This figure "fig1-8.png" is available in "png" format from: http://arxiv.org/ps/nucl-th/9410014v2 
This figure "fig1-9.png" is available in "png" format from: http://arxiv.org/ps/nucl-th/9410014v2 
This figure "fig1-10.png" is available in "png" format from: http://arxiv.org/ps/nucl-th/9410014v2 


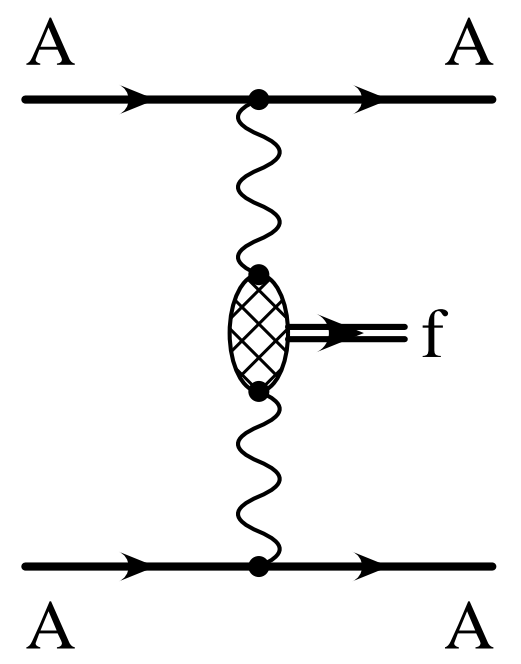

Figure 1 


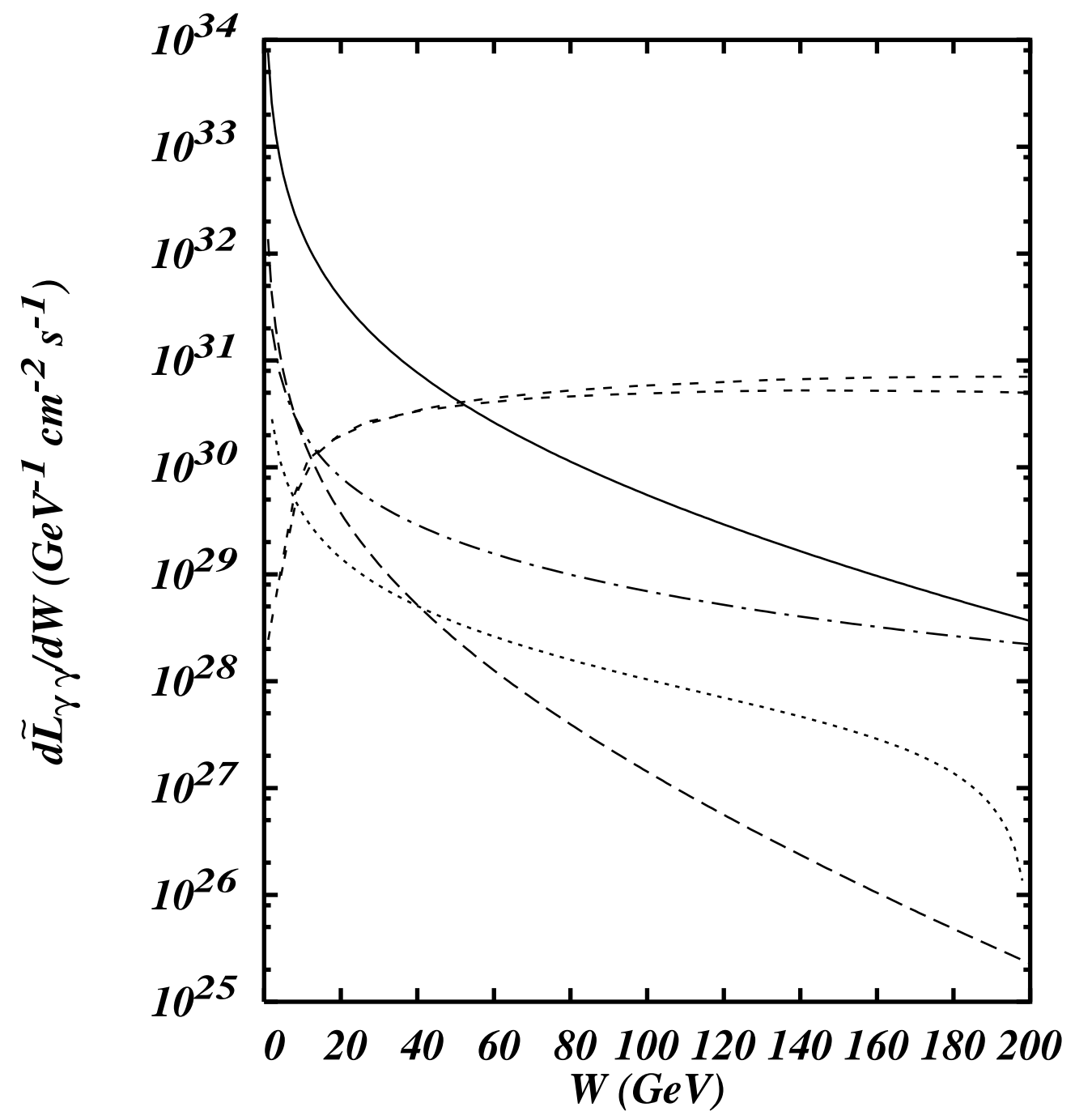

Figure 2 


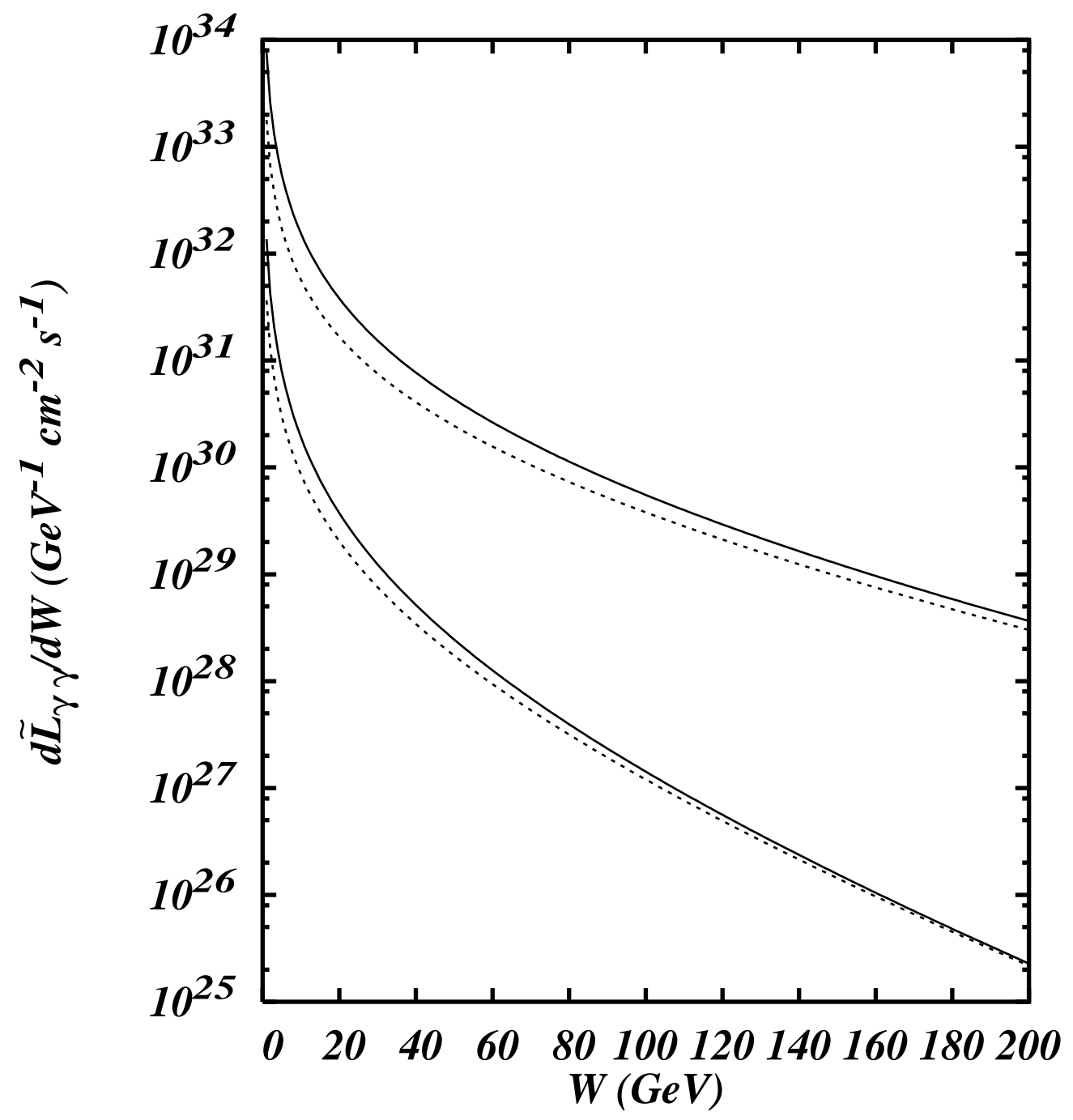

Figure 3 

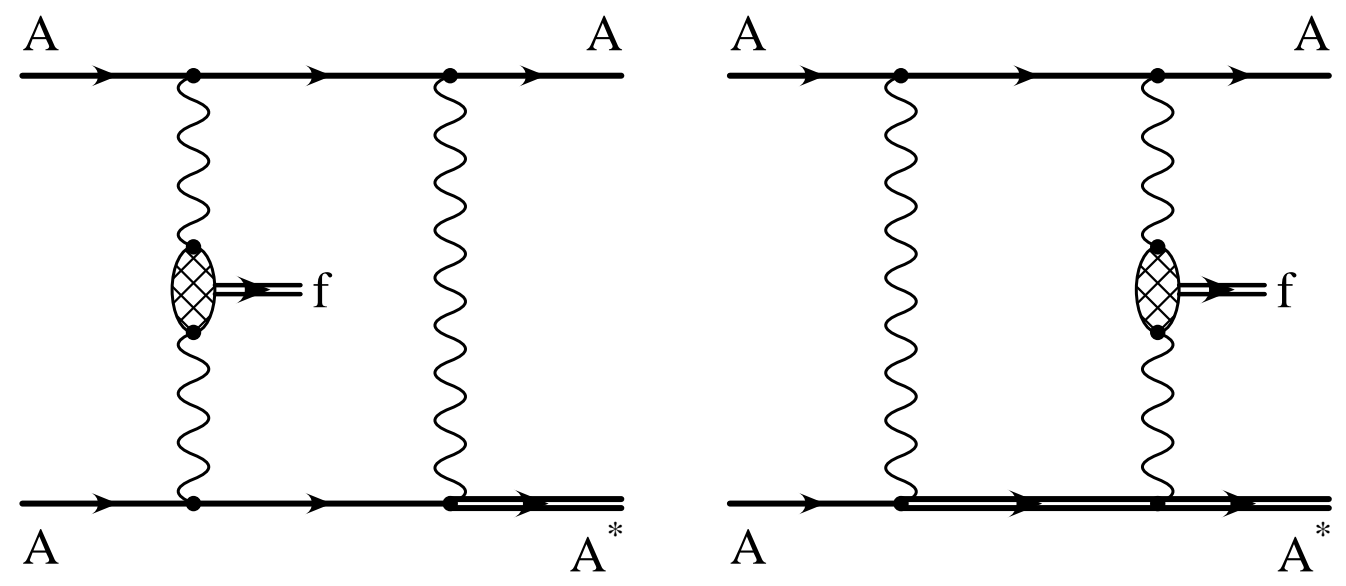

Figure 4 


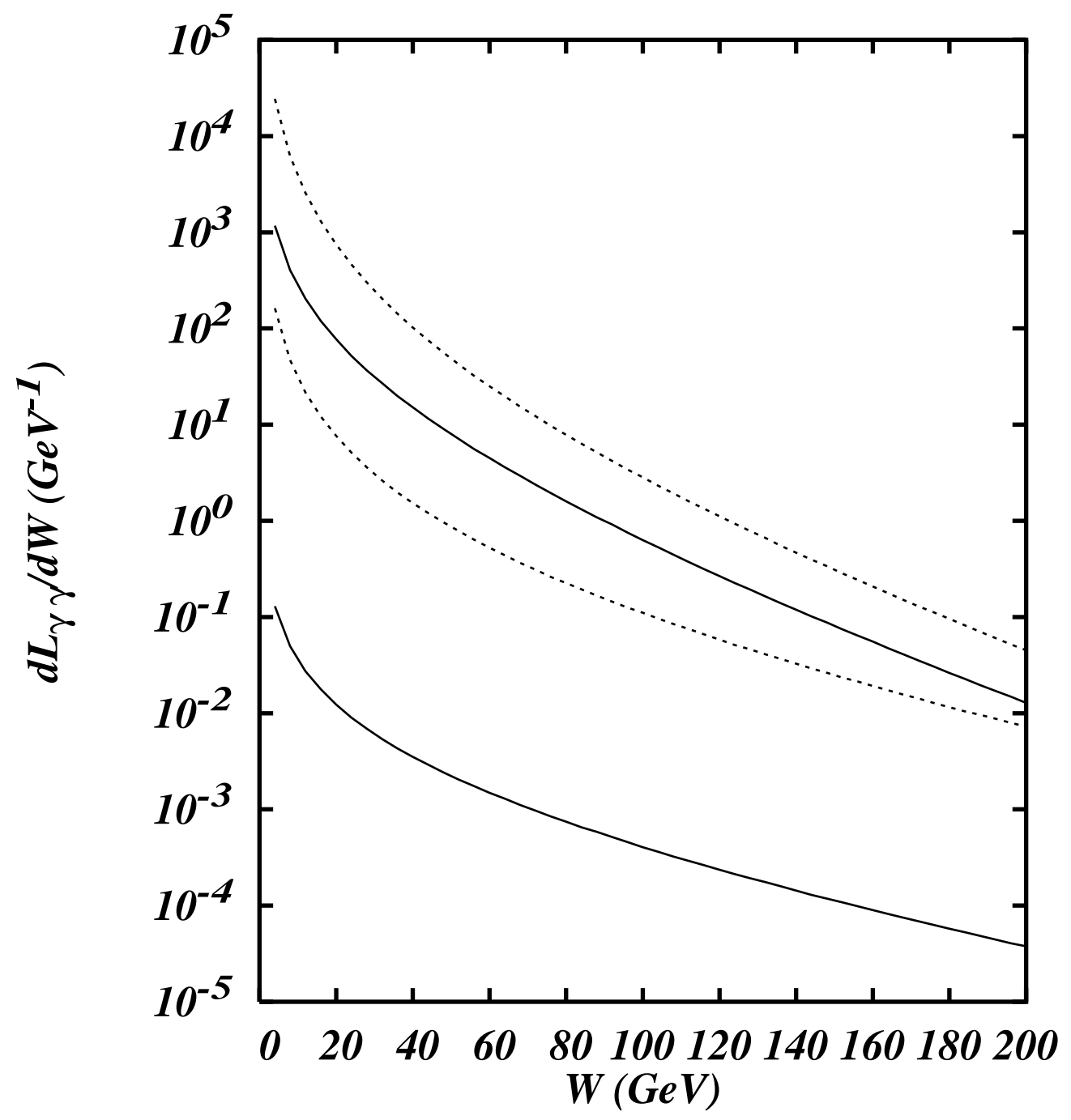

Figure 5 


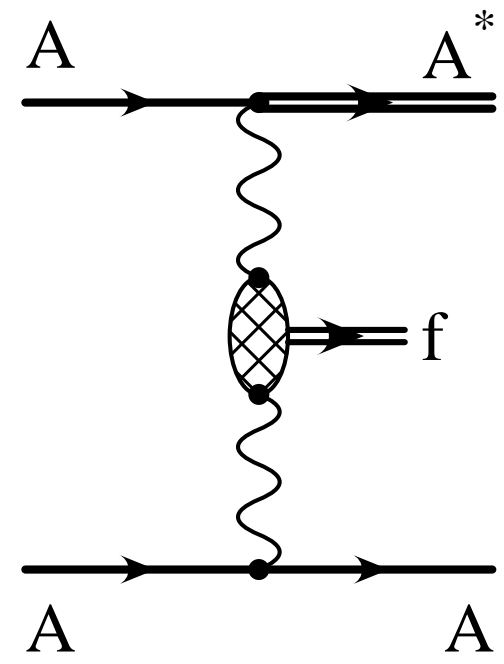

Figure 6 


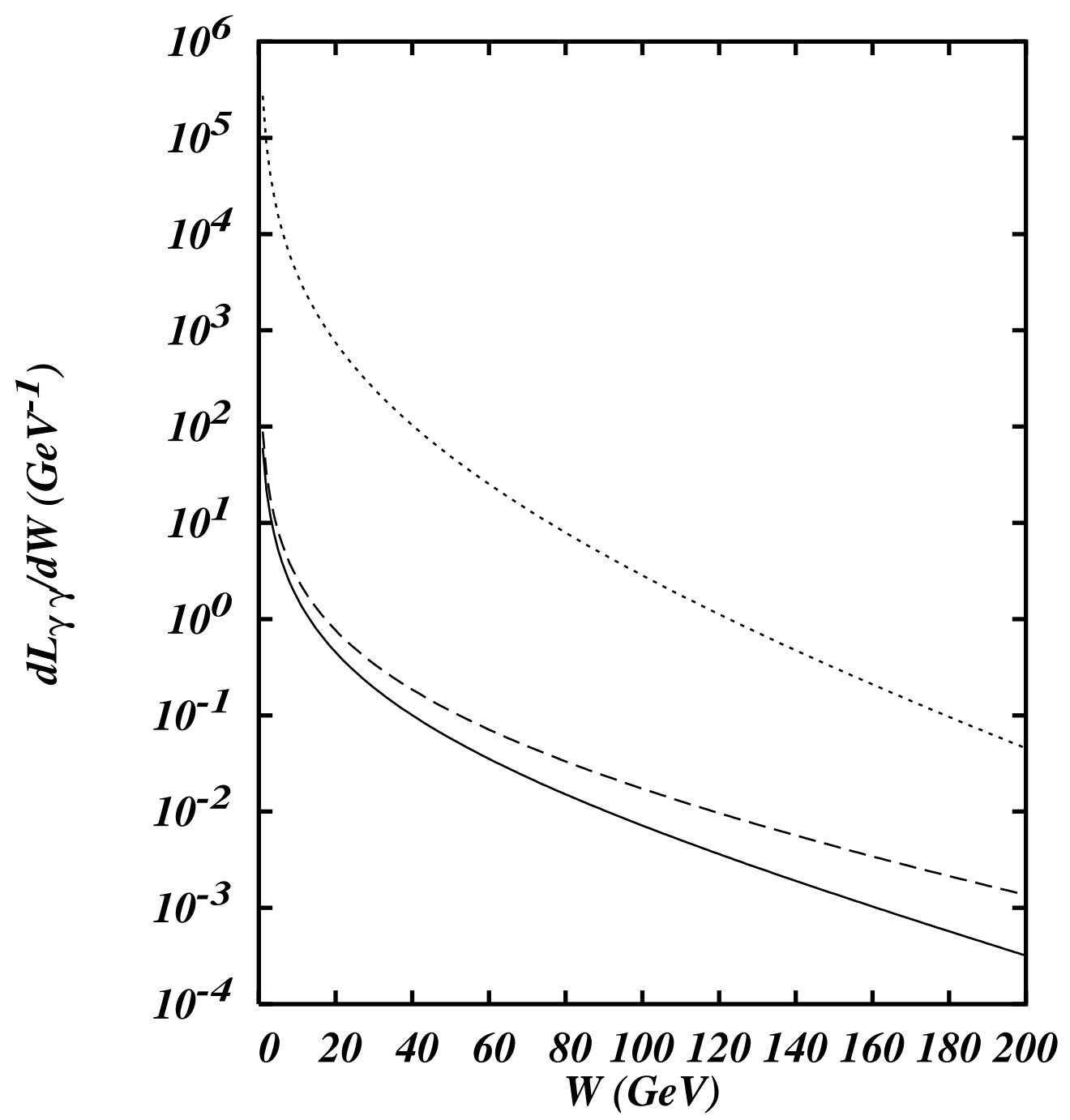

Figure 7 


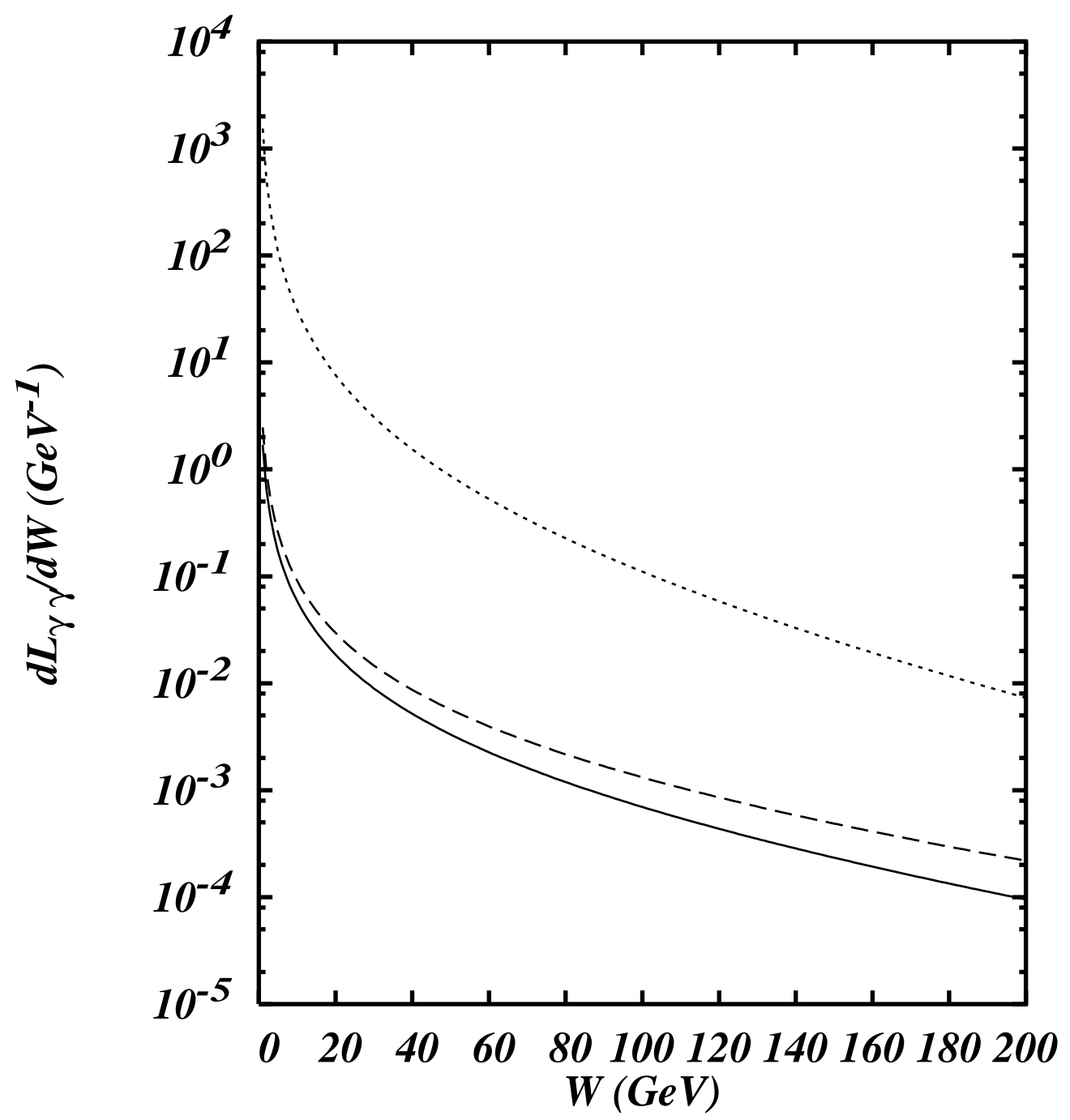

Figure 8 


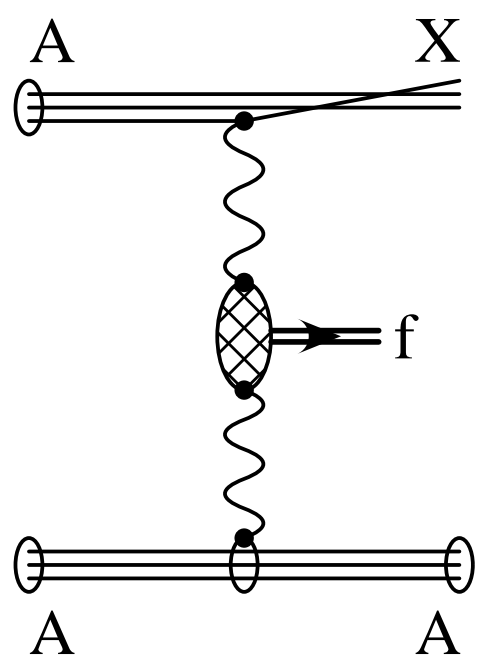

Figure 9 


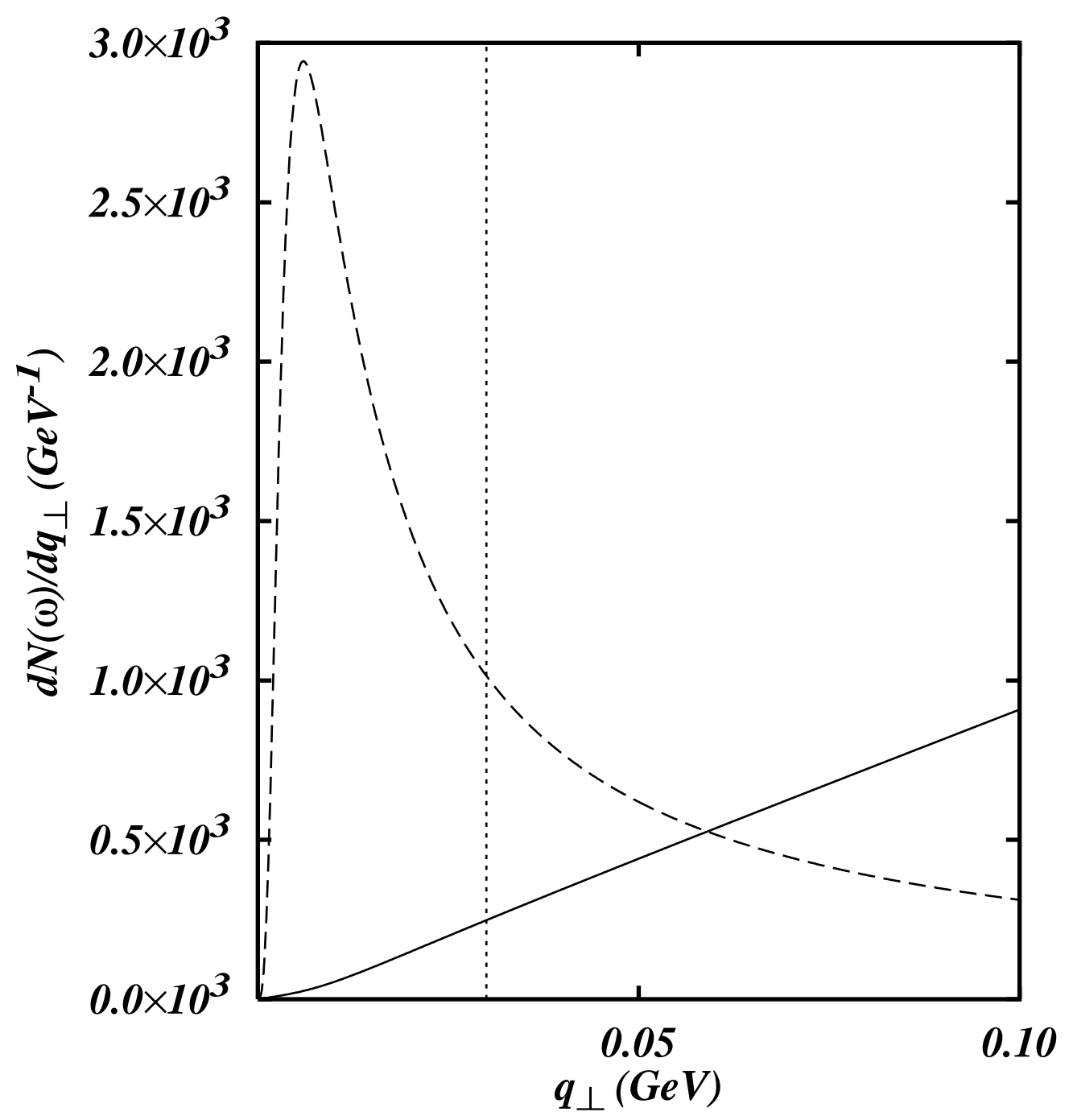

Figure 10 